\title{
Solute Mass Exchange Between Water Phase and Biofilm for a Single Pore
}

\author{
C. Z. Qin ${ }^{1}$ - S. M. Hassanizadeh ${ }^{1}$
}

Received: 2 October 2014 / Accepted: 15 May 2015 / Published online: 30 May 2015

(C) The Author(s) 2015. This article is published with open access at Springerlink.com

\begin{abstract}
Currently, there are no tractable approaches available for modeling nonequilibrium mass exchange of a solute between water phase and biofilm in porous media. The present work contributes to a quantitative description of the mass exchange of a solute over a single pore domain under a wide range of prevailing conditions. First, we developed a semiempirical model for the rate of solute mass exchange between water phase and biofilm. Then, extensive microscale simulations in a single pore were conducted. Results were averaged over a single pore domain, in order to determine a tube-scale kinetic rate coefficient as a function of various transport and biofilm properties. We illustrated the dependencies of the coefficient on a number of variables like Péclet number, Damköhler number, and biofilm volume fraction. Based on those results, we developed empirical formulae for the tube-scale mass exchange coefficient as a function of Damköhler number and biofilm volume fraction. Finally, we verified the proposed mass exchange rate against microscale simulations of solute transport in a long capillary tube. Good match was obtained over a wide range of conditions.
\end{abstract}

Keywords Porous media with biofilm · Non-equilibrium condition · Pore-scale modeling $\cdot$ Mass exchange coefficient $\cdot$ Solute transport

\section{List of symbols}

\section{Latin symbols}

$C_{\mathrm{b}}, C_{\mathrm{w}} \quad$ Microscale solute concentrations in water phase and biofilm $\left(\mathrm{M} / \mathrm{L}^{3}\right)$

C.Z. Qin

c.qin@uu.nl

1 Department of Earth Sciences, Faculty of Geosciences, University of Utrecht, P.O. Box 80021, Utrecht, The Netherlands 


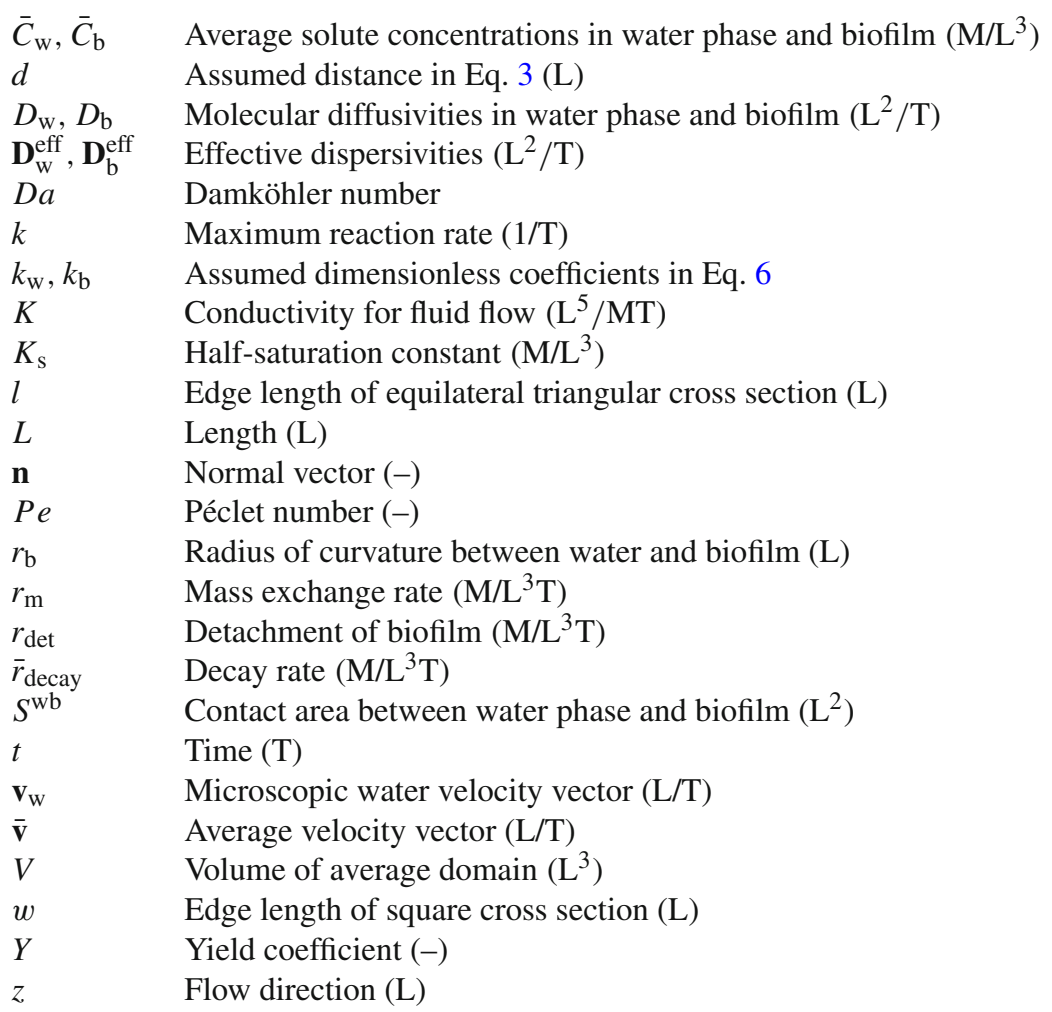

\section{Greek symbols}

$\begin{array}{ll}\varepsilon & \text { Volume fraction }(-) \\ \gamma & \text { Specific surface area (1/L) } \\ \rho & \text { Mass density }\left(\mathrm{M} / \mathrm{L}^{3}\right) \\ \varsigma & \text { Defined in Eq. } 7 \\ \xi & \text { Mass exchange coefficient (1/L) } \\ \xi^{*} & \text { Dimensionless mass exchange coefficient }(-) \\ \Gamma & \text { Diffusivity ratio (-) } \\ \mu & \text { Dynamic viscosity (M/LT) }\end{array}$

\section{Superscripts and subscripts}

$\begin{array}{ll}* & \text { Dimensionless } \\ \text { b } & \text { Biofilm } \\ \text { w } & \text { Water phase } \\ \text { ws } & \text { Water-solid interface } \\ \text { wb } & \text { Water-biofilm interface } \\ \text { bs } & \text { Biofilm-solid interface } \\ \text { ref } & \text { Reference } \\ \text { in } & \text { Inlet }\end{array}$




\section{Introduction}

In natural environments, bacteria and other microorganisms tend to attach onto soil grain surfaces. Attached bacterial cells are often embedded within a self-produced matrix of extracellular polymeric substance (EPS) which protects the cells from environmentally harsh conditions (Taylor and Jaffé 1990a, b; Ebigbo et al. 2010, 2012). These cells together with the surrounding EPS are referred to as biofilm (Iltis et al. 2011). Biofilm has been of interest to many researchers, because in the presence of water and necessary nutrients, it can grow in porous media. Biofilm occupies void pore spaces blocking water flow, which consequently reduces hydrodynamic properties of porous media like porosity and permeability. This leads to a condition known as bioclogging (see Baveye et al. 1998). In addition, biofilm has the intrinsic ability of degrading certain compounds (Wood and Whitaker 1998). So, over the past several decades, the features of bioclogging and biodegradation in porous media with biofilm have given rise to a broad range of applications, such as bioremediation, biobarriers (Baveye et al. 1998; Cunningham et al. 1991; Mitchell et al. 2009), microbial enhanced oil recovery (Afrapoli et al. 2011), and protection of steel corrosion (Videla and Herrera 2009; Zuo 2007).

A number of macroscale models for describing solute transport in porous media with biofilm are available in the literature (Baveye and Valocchi 1989; Cunningham and MendozaSanchez 2006; van Noorden et al. 2010; Ebigbo et al. 2010, 2012; Kapellos et al. 2007; Rittmann 1993; Thullner et al. 2004). There are two major classes of macroscale models: one-equation and two-equation models. Under the condition of local mass equilibrium, where the average concentrations of solute in water phase and biofilm are assumed to be linearly related, a one-equation model may be developed (Golfier et al. 2009). Under nonequilibrium conditions, where there may be mass-transfer limitation, a so-called biofilm model has been widely used (see, e.g., MacDonald et al. 1999; Rittmann 1993). Orgogozo et al. (2010) derived two one-equation models for the cases of mass-transfer limited consumption and reaction-rate limited consumption. Alternatively, under non-equilibrium conditions, a general two-equation model (one equation for solute transport in water phase, the other for solute transport in biofilm) may be used. But, the solute mass exchange between the two domains needs to be determined, for instance, by the first-order kinetics (Ebigbo et al. 2010).

In another line of research, pore-scale modeling has been employed in the studies of reactive transport in porous media with biofilm (Dupin et al. 2001; Pintelon et al. 2009). It not only sheds light on physical fundamentals of flow and transport at the pore scale, but also can provide constitutive relationships which appear in upscaled macroscale transport equations, like effective dispersive tensor, effective reaction rate, and relationship between permeability and biofilm volume fraction (Ezeuko et al. 2011; Graf von der Schulenburg et al. 2009; Hesse et al. 2009; Kim and Fogler 2000; Li et al. 2006; Stewart and Kim 2004; Suchomel et al. 1998a, b; Thullner et al. 2002; Thullner and Baveye 2008). Graf von der Schulenburg et al. (2009) developed a Lattice-Boltzmann (LB) model to study the coupled interactions between nutrient transport, biofilm growth, and hydrodynamics. The biofilm growth was described by an individual-based biofilm model which was able to track the distribution and spreading pattern of biofilm based on some local evolution rules. There are also a few studies of bioclogging in porous media using pore-network modeling (PNM). In most of these studies, only regularly arranged cylindrical tubes were used to represent the porous media of interest. Biofilm growth was assumed to occur uniformly around the pore walls. Inside a cylindrical pore, either local mass equilibrium (Ezeuko et al. 2011) or a first-order kinetic model with a constant mass exchange coefficient was used (e.g., Thullner 
and Baveye 2008). But, it is worth noting that even in the tubes of a pore network we still need to distinguish non-equilibrium from equilibrium conditions. A constant mass exchange coefficient may not capture the complex kinetics of solute transport between water phase and biofilm over a wide range of prevailing conditions. Its value is expected to be dependent on conditions of water flow, reaction rate, etc. The main objective of this work is to explore such dependencies in a single pore domain.

In this work, with the ultimate goal of developing a pore-network model of biofilm growth, we simulated solute transport in a single pore and developed relationships for tube-scale rate of solute mass exchange between water phase and biofilm. This will serve as important inputs to the PNM in future studies. The procedure is similar to the work of Raoof and Hassanizadeh (2010), Raoof et al. (2010), who developed a pore-network model of adsorbing solute transport. To avoid possible confusion, we define three scales, namely microscale, tube scale, and REV scale (or macroscale). Because micro- and REV scales are well known, we only explain tube scale as follows. At the tube scale, all quantities are defined as averages over the whole tube, such as average solute concentration or biofilm volume fraction. At the microscale, the solute mass exchange is described directly by imposing continuity of solute flux across the interface between water phase and biofilm. But, at the tube scale, a kinetic mass exchange model may be needed to couple the average concentrations in water phase and biofilm under non-equilibrium conditions. In this work, we developed this concept and obtained relationships for the tube-scale exchange rate coefficient as a function of Damköhler number and biofilm volume fraction.

The paper is organized as follows. In Sect. 2, we provide a microscale description of solute transport in pore spaces with biofilm. Then, upscaled rate of solute mass exchange between water phase and biofilm, averaged over a single pore, is presented. In Sect. 3, we develop a linear formula for the rate of kinetic mass exchange. As the first step, we investigate coefficient of this mass exchange formula for a single pore. The details of numerical experiments for determining this coefficient are presented in Sect. 4. Dependencies of tube-scale mass exchange coefficient on a number of tube-scale parameters (such as pore dimension and shape, Damköhler number, and biofilm volume fraction) are presented and discussed in Sect. 5. Also, verification studies of the proposed mass exchange rate in a long capillary tube are given. Finally, conclusions and future perspectives are provided in Sect. 6.

\section{Microscale Governing Equations of Solute Transport}

In this work, we consider saturated water flow in porous media with biofilm growing on the pore walls. The effect of planktonic biomass on solute consumption in water phase is neglected. Biofilm is treated as a continuum phase. Moreover, it is assumed that water flow through biofilm is negligible (Orgogozo et al. 2010). Therefore, with respect to solute transport, both advective and diffusive processes happen in water phase, while in biofilm only diffusion is assumed to occur. The degradation of solute in biofilm is modeled by Monod kinetics (Monod 1949). In the following, we present microscale solute transport equations. Given a rate-limiting solute, its microscale transport equations and associated boundary conditions are given as:

$$
\begin{aligned}
& \text { In the water: } \frac{\partial C_{\mathrm{w}}}{\partial t}+\nabla \cdot\left(C_{\mathrm{w}} \mathbf{v}_{\mathrm{w}}\right)-\nabla \cdot\left(D_{\mathrm{w}} \nabla C_{\mathrm{w}}\right)=0 \\
& \text { In the biofilm: } \frac{\partial C_{\mathrm{b}}}{\partial t}-\nabla \cdot\left(D_{\mathrm{b}} \nabla C_{\mathrm{b}}\right)=-k \frac{C_{\mathrm{b}}}{K_{\mathrm{s}}+C_{\mathrm{b}}} \rho_{\mathrm{b}}
\end{aligned}
$$


At the solid wall: $D_{\mathrm{w}} \nabla C_{\mathrm{w}} \cdot \mathbf{n}^{\mathrm{ws}}=D_{\mathrm{b}} \nabla C_{\mathrm{b}} \cdot \mathbf{n}^{\mathrm{bs}}=0$

At the interface between water and biofilm: $D_{\mathrm{w}} \nabla C_{\mathrm{w}} \cdot \mathbf{n}^{\mathrm{wb}}=D_{\mathrm{b}} \nabla C_{\mathrm{b}} \cdot \mathbf{n}^{\mathrm{wb}}$

where $\mathbf{v}_{\mathrm{w}}(\mathrm{L} / \mathrm{T})$ is the water velocity, $C_{\mathrm{w}}\left(\mathrm{M} / \mathrm{L}^{3}\right)$ is the mass concentration of solute in the water, $C_{\mathrm{b}}\left(\mathrm{M} / \mathrm{L}^{3}\right)$ is the mass concentration of solute in the biofilm, $D_{\mathrm{w}}$ and $D_{\mathrm{b}}\left(\mathrm{L}^{2} / \mathrm{T}\right)$ are the molecule diffusivities of solute in the water and biofilm, respectively, $\mathbf{n}^{\mathrm{ws}}$ and $\mathbf{n}^{\mathrm{bs}}$ are the normal vectors pointing to the solid wall, $\mathbf{n}^{\mathrm{wb}}$ is the normal vector outward from the water phase, $k(1 / \mathrm{T})$ is the maximum reaction rate, $\rho_{\mathrm{b}}\left(\mathrm{M} / \mathrm{L}^{3}\right)$ is the biomass density (dry mass per wet volume), and $K_{\mathrm{S}}\left(\mathrm{M} / \mathrm{L}^{3}\right)$ is the half-saturation constant at which the reaction rate is half of $k \rho_{\mathrm{b}}$.

To obtain the upscaled rate of solute mass exchange between water phase and biofilm over a single pore, the volume-averaging technique (Hassanizadeh and Gray 1979; Wood and Whitaker 1998, 2000; Davit et al. 2010) is applied to the above microscale transport equations. The details are given in "Appendix 1." The resulting formula for average mass exchange rate is given as:

$$
r_{\mathrm{m}}=-D_{\mathrm{w}} \frac{1}{V} \int_{S^{\mathrm{wb}}} \nabla C_{\mathrm{w}} \cdot \mathbf{n}^{\mathrm{wb}} \mathrm{d} \sigma=D_{\mathrm{b}} \frac{1}{V} \int_{S^{\mathrm{wb}}} \nabla C_{\mathrm{b}} \cdot \mathbf{n}^{\mathrm{bw}} \mathrm{d} \sigma
$$

Here, $S^{\mathrm{wb}}$ denotes the boundary between water and biofilm domains within a single pore, and $r_{\mathrm{m}}\left(\mathrm{M} / \mathrm{L}^{3} \mathrm{~T}\right)$ denotes the rate of solute mass exchange between the two domains.

As seen in Eq. 2, the current form of solute mass exchange is still related to the microscale information on solute concentration gradients. At the tube scale, the mass exchange term may be expressed as a function of the average solute concentrations in the two domains because of the selection of primary variables in the governing equations. Such a relationship is developed in the next section. It must be noted that the upscaled solute transport equations (35 and 37) are valid for any averaging domain. That can be, for example, a long capillary tube, a bundle of tubes, or a REV. The form of equations remains the same, only the interpretation of average-scale variables will be different. The same holds for the empirical model of mass exchange rate developed in the next section.

\section{Rate of Solute Mass Exchange Between Water Phase and Biofilm}

As described in the previous section, the volume-averaged rate of solute mass exchange between water phase and biofilm is given by Eq. 2. At the microscale, solute concentration gradient at the wb interface can be approximated in the following manner (see Raoof and Hassanizadeh 2010):

$$
\begin{aligned}
& \text { In the water: } \nabla C_{\mathrm{w}} \cdot \mathbf{n}^{\mathrm{wb}}=\frac{\left.C_{\mathrm{w}}\right|_{\mathrm{wb}}-\left.C_{\mathrm{w}}\right|_{\text {local }}}{d} \\
& \text { In the biofilm: } \nabla C_{\mathrm{b}} \cdot \mathbf{n}^{\mathrm{wb}}=\frac{\left.C_{\mathrm{b}}\right|_{\text {local }}-\left.C_{\mathrm{b}}\right|_{\mathrm{wb}}}{d}
\end{aligned}
$$

Here, the subscript local indicates that the solute concentration is evaluated at a point within water or biofilm domains located at a distance $d$ from the interface, and the subscript wb indicates the solute concentration at the interface. Then, substitution of Eqs. $3 \mathrm{a}$ and $3 \mathrm{~b}$ into Eq. 2 yields:

$$
r_{\mathrm{m}}=-D_{\mathrm{w}} \frac{1}{V} \int_{S^{\mathrm{wb}}} \frac{\left.C_{\mathrm{w}}\right|_{\mathrm{wb}}-\left.C_{\mathrm{w}}\right|_{\text {local }}}{d} \mathrm{~d} \sigma=-D_{\mathrm{b}} \frac{1}{V} \int_{S^{\mathrm{wb}}} \frac{\left.C_{\mathrm{b}}\right|_{\text {local }}-\left.C_{\mathrm{b}}\right|_{\mathrm{wb}}}{d} \mathrm{~d} \sigma
$$


We assume the continuity of solute concentration at the interface, i.e., $\left.C_{\mathrm{w}}\right|_{\mathrm{wb}}=\left.C_{\mathrm{b}}\right|_{\mathrm{wb}}$. So, we can eliminate this term between the two integrals in Eq. 4, and after some algebra, we obtain the following expression for the mass exchange rate:

$$
r_{\mathrm{m}}=\frac{D_{\mathrm{w}} D_{\mathrm{b}}}{D_{\mathrm{w}}+D_{\mathrm{b}}} \frac{1}{V} \frac{1}{d} \int_{S^{\mathrm{wb}}}\left(\left.C_{\mathrm{w}}\right|_{\text {local }}-\left.C_{\mathrm{b}}\right|_{\text {local }}\right) \mathrm{d} \sigma
$$

Note that the diffusivity product appears in the numerator. The mass exchange would vanish if either of diffusivity coefficients goes to zero. Now, in an empirical way, we linearly relate the integral of those microscale concentrations to the average concentrations by introducing two variable coefficients:

$$
\begin{gathered}
\left.\int_{S^{\mathrm{wb}}} C_{\mathrm{w}}\right|_{\text {local }} \mathrm{d} \sigma=k_{\mathrm{w}} S^{\mathrm{wb}} \bar{C}_{\mathrm{w}} \\
\left.\int_{S^{\mathrm{wb}}} C_{\mathrm{b}}\right|_{\text {local }} \mathrm{d} \sigma=k_{\mathrm{b}} S^{\mathrm{wb}} \bar{C}_{\mathrm{b}}
\end{gathered}
$$

where $k_{\mathrm{w}}$ and $k_{\mathrm{b}}$ are the postulated dimensionless coefficients which may depend on flow conditions in a single pore.

Substitution of Eqs. 6a and 6b into Eq. 5 and redefining variables yield:

$$
r_{\mathrm{m}}=\frac{D_{\mathrm{w}} D_{\mathrm{b}}}{D_{\mathrm{w}}+D_{\mathrm{b}}} \gamma \xi\left(\bar{C}_{\mathrm{w}}-\varsigma \bar{C}_{\mathrm{b}}\right)
$$

where

$$
\gamma=\frac{S^{\mathrm{wb}}}{V}(1 / \mathrm{L}), \quad \xi=\frac{k_{\mathrm{w}}}{d}(1 / \mathrm{L}), \quad \varsigma=\frac{k_{\mathrm{b}}}{k_{\mathrm{w}}}(-)
$$

Here, $\gamma$ is called the specific surface area of biofilm over the pore domain. The idealized case is to fit both coefficients for a single pore. However, for simplicity, in our following calculations, we only focus on the first coefficient $\xi$ by setting $\varsigma$ to unity. Thus, the mass exchange rate is simplified as:

$$
r_{\mathrm{m}}=\frac{D_{\mathrm{w}} D_{\mathrm{b}}}{D_{\mathrm{w}}+D_{\mathrm{b}}} \gamma \xi\left(\bar{C}_{\mathrm{w}}-\bar{C}_{\mathrm{b}}\right)
$$

At this point, we have derived the empirical mass exchange rate of solute (Eq. 9). It is quite similar to the traditionally used linear exchange term, except that two solute diffusivities are explicitly included in our formula. Note that in the following, we will refer to the coefficient $\xi$ as the mass exchange coefficient.

\section{Numerical Determination of Mass Exchange Coefficient $\xi$ for a Single Pore}

In this section, we describe the numerical procedure for determining the mass exchange coefficient $\xi$. First, microscale equations (1a-1d) are made dimensionless and then solved numerically in a single pore domain. From the numerical solution, average concentrations for the whole water and biofilm domains, as well as the mass exchange rate, $r_{\mathrm{m}}$, are calculated. Results are substituted back into Eq. 9 in order to calculate $\xi$. We considered three typical shapes of pore cross sections. These are circle, square, and equilateral triangle as shown in Fig. 1. We note that the biofilm accumulation pattern in a pore depends on the shape of its cross section and many other factors. In the present work, we defined the following 
(a)
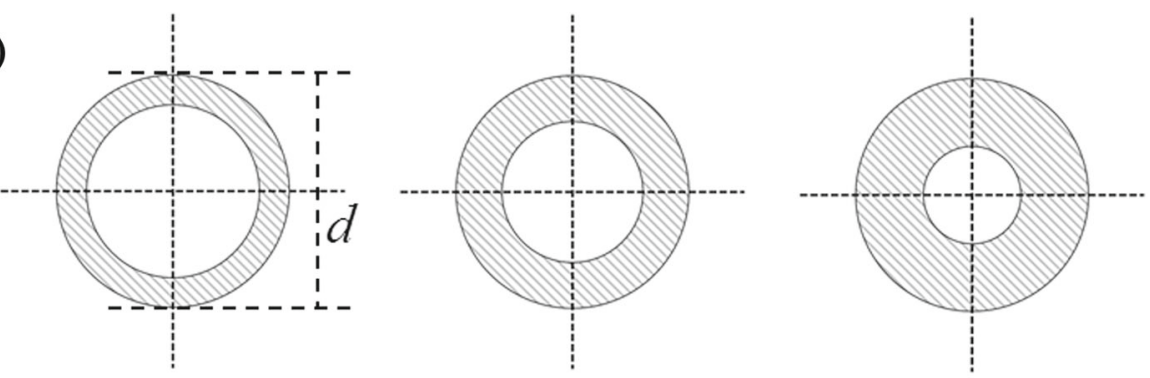

(b)
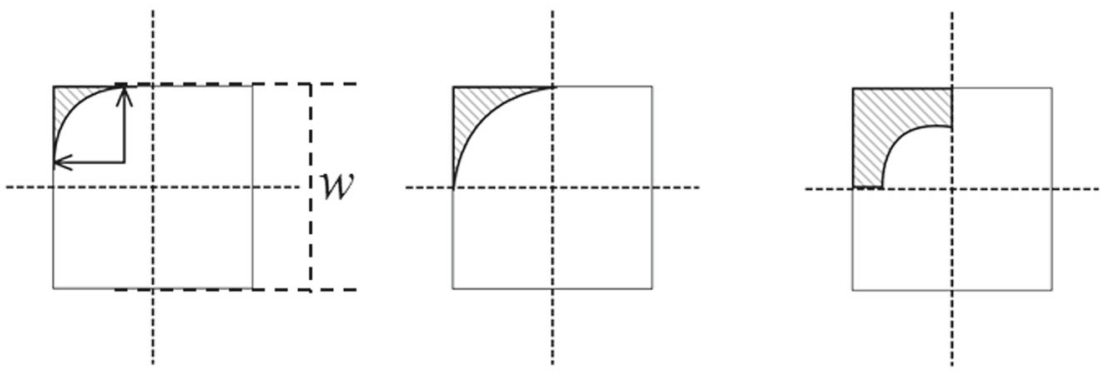

(c)
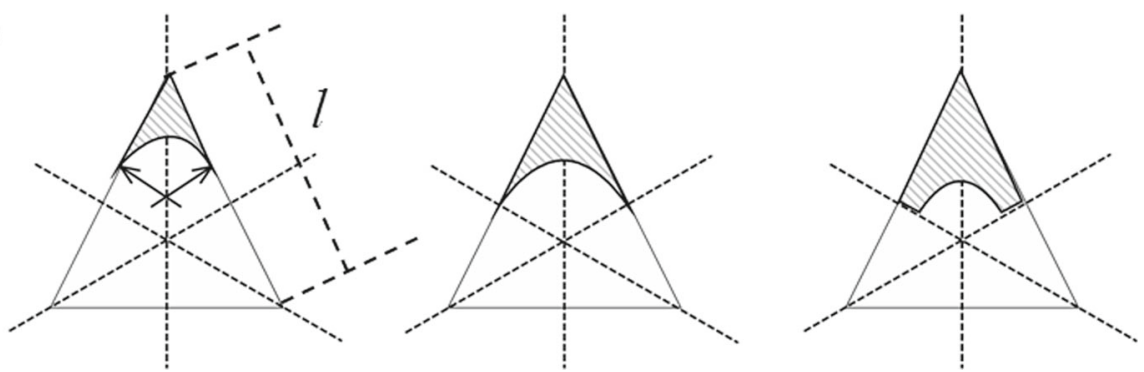

Fig. 1 Three types of pore cross section. The growth pattern of biofilm and its accumulation within the pore are indicated from left to right (notice that only one corner is schematically shown in the case of square or equilateral triangle)

prespecified accumulation patterns for the three different cross sections. In the case of circle, biofilm is assumed to attach to the pore walls with a uniform thickness (see Fig. 1a). In the case of square and equilateral triangle, biofilm is assumed to grow in the corners, as shown in Fig. 1b, c.

\subsection{Dimensionless Microscopic Equations of Solute Transport}

For making microscale equations (1a and 1b) dimensionless, we define the following dimensionless variables (denoted by an asterisk) and numbers:

$$
\begin{gathered}
C_{\mathrm{w}}^{*}=\frac{C_{\mathrm{w}}}{K_{\mathrm{s}}}, \quad C_{\mathrm{b}}^{*}=\frac{C_{\mathrm{b}}}{K_{\mathrm{s}}}, \quad P e^{\mathrm{w}}=\frac{\bar{v}_{\mathrm{z}} L^{\mathrm{ref}}}{D_{\mathrm{w}}}, \quad P e=\frac{v^{\mathrm{ref}} L^{\mathrm{ref}}}{D_{\mathrm{w}}}, \\
\Gamma=\frac{D_{\mathrm{b}}}{D_{\mathrm{w}}}, \quad t^{*}=\frac{D_{\mathrm{w}} t}{\left(L^{\mathrm{ref}}\right)^{2}}, \quad D a=\frac{k \rho_{\mathrm{b}}}{K_{\mathrm{s}} D_{\mathrm{w}}}\left(L^{\mathrm{ref}}\right)^{2}
\end{gathered}
$$


Here, the half-saturation constant, $K_{\mathrm{S}}$, is selected to be the reference concentration, $L^{\text {ref }}$ is the reference length, $\bar{v}_{\mathrm{z}}$ is the average water velocity in the pore, $v^{\text {ref }}$ is the reference velocity, $P \mathrm{e}^{\mathrm{w}}$ and $P$ e are the tube-scale and microscale Péclet numbers in the water phase, respectively, $\Gamma$ is the diffusivity ratio, and $D a$ is Damköhler number defined as the ratio of reaction rate to diffusive mass-transfer rate. For different cross sections, we select different reference lengths which are $d, w$, and $l$ for circle, square, and equilateral triangle, respectively (see Fig. 1). In the following, the dimensionless solute transport equations in a general pore will be presented associated with the velocity field along the flow direction.

For a pore with non-circular cross section, we need to numerically obtain the $z$-velocity distribution. Toward this end, the Stokes equation (assuming no transversal velocities) is solved. In addition, it is assumed that the pressure gradient along the tube is constant:

$$
\nabla^{2} v_{\mathrm{z}}=\frac{1}{\mu} \frac{\mathrm{d} p}{\mathrm{~d} z}=\text { constant }
$$

No-slip condition is assumed at the pore wall and water-biofilm interfaces: $v_{\mathrm{Z}}=0$

To make Eq. 11 dimensionless, we employ the following dimensionless variables:

$$
\begin{aligned}
z^{*} & =\frac{z}{L^{\text {ref }}}, \quad \nabla^{*}=L^{\text {ref }} \nabla \\
v_{\mathrm{z}}^{*} & =\frac{v_{\mathrm{z}}}{v^{\text {ref }}}\left(v^{\text {ref }}=-\frac{\left(L^{\text {ref }}\right)^{2}}{\mu} \frac{\mathrm{d} p}{\mathrm{~d} z}, \text { the selected reference velocity }\right)
\end{aligned}
$$

Then, the dimensionless Stokes equation reads:

$$
\nabla^{* 2} v_{\mathrm{z}}^{*}=-1
$$

Based on the numerically obtained $z$-velocity distribution, the conductivity of the pore can be calculated as:

$$
K=\frac{\int_{A} v_{\mathrm{z}} \mathrm{d} \sigma}{-\mathrm{d} p / \mathrm{d} z}=\frac{\left(L^{\mathrm{ref}}\right)^{4}}{\mu} \int_{A^{*}} v_{\mathrm{z}}^{*} \mathrm{~d} \sigma^{*}=\frac{\left(L^{\mathrm{ref}}\right)^{4}}{\mu} K^{*}
$$

where $A$ is the cross-sectional area available for water flow and $K^{*}=\int_{A^{*}} v_{\mathrm{Z}}^{*} \mathrm{~d} \sigma^{*}$ is the dimensionless conductivity. Its value would depend on the biofilm volume fraction.

Now, according to the dimensionless variables listed in (10) and (12), the dimensionless equations of solute transport can be written as:

$$
\begin{aligned}
& \text { In the water phase: } \frac{\partial C_{\mathrm{w}}^{*}}{\partial t^{*}}+P e v_{\mathrm{z}}^{*} \frac{\partial C_{\mathrm{w}}^{*}}{\partial z^{*}}-\nabla^{* 2} C_{\mathrm{w}}^{*}=0 \\
& \text { In the biofilm: } \frac{\partial C_{\mathrm{b}}^{*}}{\partial t^{*}}-\Gamma \nabla^{* 2} C_{\mathrm{b}}^{*}+D a \frac{C_{\mathrm{b}}^{*}}{1+C_{\mathrm{b}}^{*}}=0
\end{aligned}
$$

Based on the geometric information and assumed biofilm evolution pattern in each type of pore domain (see Fig. 2), we obtain the following geometric relationships for specific cross sections:

1. Circle (Fig. 2a):

$$
\begin{aligned}
r_{\mathrm{b}}^{*} & =0.5 \sqrt{1-\varepsilon^{\mathrm{b}}} \\
S_{\mathrm{wb}}^{*} & =\pi L^{*} \sqrt{1-\varepsilon^{\mathrm{b}}}
\end{aligned}
$$


(a)

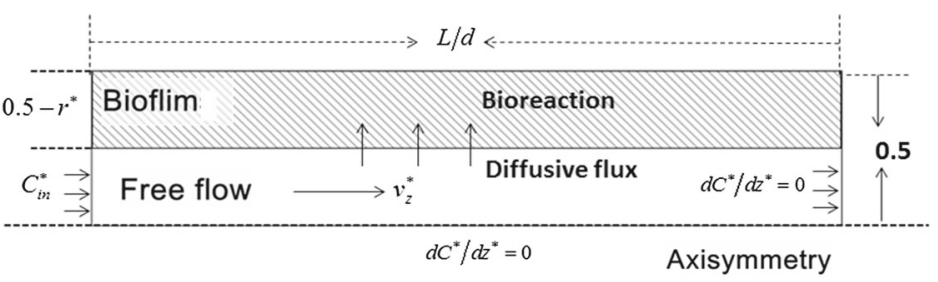

(b)

$L / w$

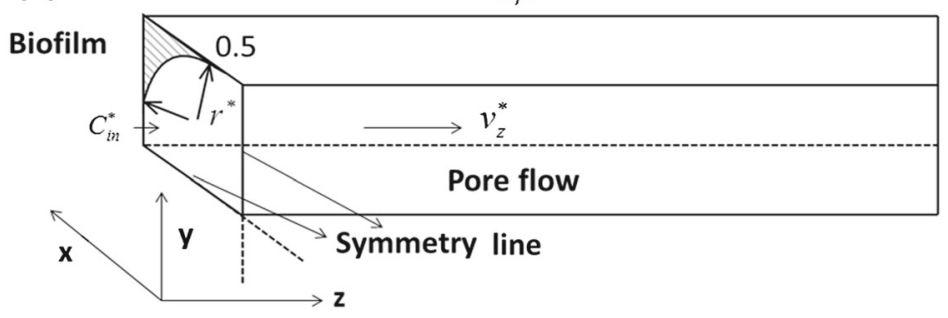

(c)

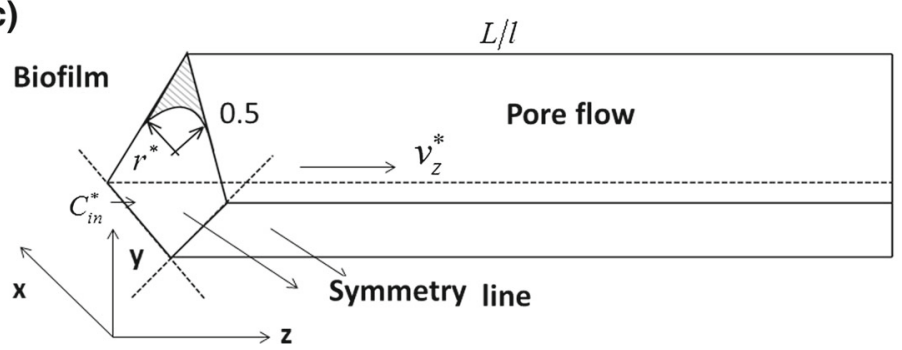

Fig. 2 Dimensionless computational domains for three pores with different cross sections in rectangular coordinates: a circle; $\mathbf{b}$ square, a quarter is shown; and $\mathbf{c}$ equilateral triangle, a corner is shown

where $r_{\mathrm{b}}^{*}$ is the dimensionless radius of curvature of the interface between the water phase and biofilm and $S_{\mathrm{wb}}^{*}$ is the dimensionless interfacial area (i.e., the contact area). Here, the original diameter of the circular cross section is taken as the reference length.

2. Square (Fig. 2b):

$$
\begin{aligned}
r_{\mathrm{b}}^{*} & = \begin{cases}\sqrt{\frac{\varepsilon^{\mathrm{b}}}{4-\pi}} & \varepsilon^{\mathrm{b}} \leq 1-\frac{\pi}{4} \\
\sqrt{\frac{1-\varepsilon^{\mathrm{b}}}{\pi}} & \varepsilon^{\mathrm{b}}>1-\frac{\pi}{4}\end{cases} \\
S_{\mathrm{wb}}^{*} & = \begin{cases}2 \pi L^{*} \sqrt{\frac{\varepsilon^{\mathrm{b}}}{4-\pi}} & \varepsilon^{\mathrm{b}} \leq 1-\frac{\pi}{4} \\
2 L^{*} \sqrt{\left(1-\varepsilon^{\mathrm{b}}\right) \pi} & \varepsilon^{\mathrm{b}}>1-\frac{\pi}{4}\end{cases}
\end{aligned}
$$

3. Equilateral triangle (Fig. 2c):

$$
r_{\mathrm{b}}^{*}= \begin{cases}\sqrt{\frac{\sqrt{3} \varepsilon^{\mathrm{b}}}{12 \sqrt{3}-4 \pi}} & \varepsilon^{\mathrm{b}} \leq 1-\frac{\sqrt{3}}{9} \pi \\ \sqrt{\frac{\sqrt{3}\left(1-\varepsilon^{\mathrm{b}}\right)}{4 \pi}} & \varepsilon^{\mathrm{b}}>1-\frac{\sqrt{3}}{9} \pi\end{cases}
$$




$$
S_{\mathrm{wb}}^{*}= \begin{cases}\pi L^{*} \sqrt{\frac{\sqrt{3} \varepsilon^{\mathrm{b}}}{3 \sqrt{3}-\pi}} & \varepsilon^{\mathrm{b}} \leq 1-\frac{\sqrt{3}}{9} \pi \\ \pi L^{*} \sqrt{\frac{\sqrt{3}\left(1-\varepsilon^{\mathrm{b}}\right)}{\pi}} & \varepsilon^{\mathrm{b}}>1-\frac{\sqrt{3}}{9} \pi\end{cases}
$$

Also, we can calculate the average water velocity in the pore as:

$$
\bar{v}_{\mathrm{z}}=v^{\mathrm{ref}} \frac{\left(L^{\mathrm{ref}}\right)^{2}}{A^{0}} \frac{K^{*}}{1-\varepsilon^{\mathrm{b}}}
$$

where $A^{0}$ is the cross-sectional area of a pore in the absence of biofilm. Then, we are able to define the pore-scale Péclet number for the whole pore as:

$$
P e^{\mathrm{w}}=\frac{\bar{v}_{\mathrm{z}} L^{\mathrm{ref}}}{D_{\mathrm{w}}}=\frac{\bar{v}_{\mathrm{z}}}{v^{\mathrm{ref}}} P e
$$

Finally, it is noted that in the case of circular cross section, the axisymmetric transport equations (47 and 48) are employed in numerical experiments. The derivation is presented in "Appendix 2."

\subsection{Dimensionless Mass Exchange Rate}

To better fit the coefficient from numerically calculated dimensionless microscale information, we relate the mass exchange rate to its dimensionless form as:

$$
r_{\mathrm{m}}=\frac{D_{\mathrm{w}} K_{\mathrm{s}}}{\left(L^{\mathrm{ref}}\right)^{2}} r_{\mathrm{m}}^{*}
$$

where

$$
r_{\mathrm{m}}^{*}=\gamma^{*} \xi^{*} \frac{\Gamma}{1+\Gamma}\left(\bar{C}_{\mathrm{w}}^{*}-\bar{C}_{\mathrm{b}}^{*}\right)
$$

Here, $\xi^{*}=\xi L^{\text {ref }}$ is the dimensionless mass exchange coefficient, $\gamma^{*}=\gamma L^{\text {ref }}$ is the dimensionless specific surface area, and $\bar{C}_{\mathrm{w}}^{*}$ and $\bar{C}_{\mathrm{b}}^{*}$ are the dimensionless average solute concentrations in the water phase and biofilm, respectively. By solving the dimensionless solute transport equations in each case, we can calculate the dimensionless mass exchange rate by:

$$
r_{\mathrm{m}}^{*}=-\left.\gamma^{*}\left(\overline{\nabla^{*} C_{\mathrm{w}}^{*}} \cdot \mathbf{n}^{\mathrm{wb}}\right)\right|_{\mathrm{wb}}
$$

where $\overline{\nabla^{*} C_{\mathrm{w}}^{*}} \cdot \mathbf{n}^{\text {wb }}$ denotes the average dimensionless solute flux over the contact area, into the biofilm.

Finally, from Eqs. 22 to 23, we get the following relationship:

$$
\xi^{*} \frac{\Gamma}{1+\Gamma}\left(\bar{C}_{\mathrm{w}}^{*}-\bar{C}_{\mathrm{b}}^{*}\right)=-\left.\left(\overline{\nabla * C_{\mathrm{w}}^{*}} \cdot \mathbf{n}^{\mathrm{wb}}\right)\right|_{\mathrm{wb}}
$$

From numerical simulation of microscale transport equations, all terms except $\xi^{*}$ in Eq. 24 can be calculated. Thus, we can determine $\xi^{*}$ for each set of solution.

\subsection{Description of Numerical Simulations}

The dimensionless microscale equations of solute transport in both water phase and biofilm were solved in a commercial software COMSOL, which is FEM (finite element method) based. It is noted that only steady-state numerical results were used for coefficient fittings. This is because the timescale for solute transport through a pore domain is much smaller than 
Table 1 Employed dimensionless parameters for the base cases

\begin{tabular}{ll}
\hline Parameter & Value \\
\hline Diffusivity ratio, $\Gamma$ & 0.1 \\
Inlet mass concentration of solute, $C_{\text {in }}^{*}$ & 10 \\
Peclet number in pore flow, $P e^{\mathrm{w}}$ & 10 \\
Damköhler number, $D a$ & 10 \\
Dimensionless length of pore throat, $L^{*}$ & 5 \\
Volume fraction of biofilms, $\varepsilon^{\mathrm{b}}$ & 0.75 for circular \\
& $1-\pi / 4$ for square \\
& $1-\sqrt{3} \pi / 9$ for equilateral triangular
\end{tabular}

that for biofilm growth. For the three different computational domains, as shown in Fig. 2, the symmetry was used for reducing computational efforts. The following boundary conditions were imposed. In the water phase, we used a constant solute concentration $C_{\text {in }}^{*}$ at the inlet and fully developed condition at the outlet; in the biofilm, no-flux boundary conditions were imposed; and the condition of continuous solute flux was used at the interface between the water flow and biofilm.

For each type of pore domain, we set up a base case for the later reference. The employed dimensionless physical and geometric parameters are listed in Table 1. In the mesh independence study, we increased and decreased the mesh density by $40 \%$, and no discernible difference in solute concentration distributions was found. In next section, we first present fitted conductivities for water flow as a function of biofilm volume fraction. Then, we investigate the dependencies of the dimensionless coefficient $\xi^{*}$ on Péclet number, biofilm volume fraction, ratio of diffusivity in biofilm to that in water, etc. Finally, we present verification studies of the proposed mass exchange rate.

\section{Results and Discussion}

\subsection{Pore Conductivity Under Biofilm Growth}

Pore conductivity is an important input parameter for the PNM (Budek and Szymczak 2012). It is expected that the conductivity for water flow decreases with the accumulation of biofilm. According to the assumed accumulation patterns of biofilm, we plotted the dimensionless conductivities versus biofilm volume fraction for the three types of pore domains in Fig. 3. In the case of circle, the dimensionless conductivity is given as:

$$
K^{*}=\frac{\pi}{128}\left(1-\varepsilon^{\mathrm{b}}\right)^{2}
$$

In the cases of square and equilateral triangle, we fitted the numerical results with polynomial expressions and obtained the following empirical formulae:

Square:

$$
\begin{aligned}
K^{*}= & 0.161\left(\varepsilon^{b}\right)^{5}-0.471\left(\varepsilon^{b}\right)^{4}+0.516\left(\varepsilon^{b}\right)^{3} \\
& -0.219\left(\varepsilon^{b}\right)^{2}-0.022 \varepsilon^{b}+0.035
\end{aligned}
$$




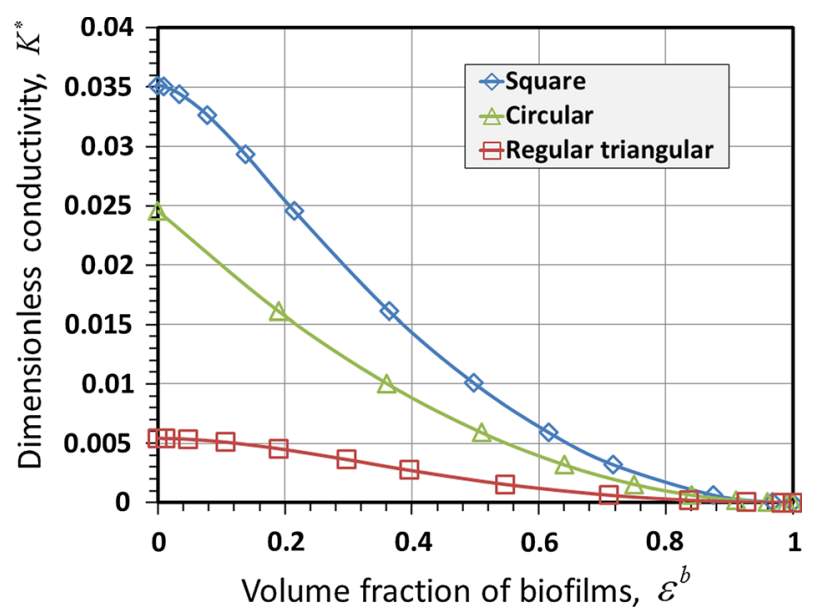

Fig. 3 Pore conductivities as a function of biofilm volume fraction

Equilateral triangle:

$$
\begin{aligned}
K^{*}= & -0.0142\left(\varepsilon^{\mathrm{b}}\right)^{4}+0.0378\left(\varepsilon^{\mathrm{b}}\right)^{3}-0.0285\left(\varepsilon^{\mathrm{b}}\right)^{2} \\
& -0.0005 \varepsilon^{\mathrm{b}}+0.0054
\end{aligned}
$$

It is seen that the conductivity of a general pore is mainly determined by the shape of its cross section and the biofilm volume fraction. We note that following the procedure used in this work, the conductivity of a pore with arbitrary shape of cross section can be numerically fitted as a function of biofilm volume fraction.

\subsection{Mass Exchange Coefficient for the Case of Circular Cross Section}

In most PNM of biofilm, usually pores are assumed to have circular cross sections (e.g., Kim and Fogler 2000; Thullner and Baveye 2008). In this subsection, results for the dependencies of the dimensionless mass exchange coefficient $\xi^{*}$ in a pore domain with circular cross section are presented. To fully understand the dependencies, we selected six dimensionless variables which may affect this coefficient. They are length of pore domain $\left(L^{*}\right)$, Péclet number $\left(P e^{\mathrm{w}}\right)$, Damköhler number $(D a)$, biofilm volume fraction $\left(\varepsilon^{\mathrm{b}}\right)$, inlet solute concentration $\left(C_{\text {in }}^{*}\right)$, and diffusivity ratio $(\Gamma)$.

Figure 4 shows the dependencies of the dimensionless mass exchange coefficient on the length of pore domain and Péclet number. To demonstrate individual dependencies, we presented the numerical results in the following way. In each graph, we plotted the dependency of $\xi^{*}$ on the variable given by the horizontal axis under the value of variable given by the legend. Meanwhile, the values of the rest four variables are kept constant given in Table 1. It is seen that $\xi^{*}$ is independent of pore length. It is also found that at low Péclet numbers $\left(P e^{\mathrm{w}}<1\right), \xi^{*}$ can be regarded as being independent of $P e^{\mathrm{w}}$. When $P e^{\mathrm{w}}>1$, there are slight variations in $\xi^{*}$ in the ranges of large Damköhler number, small biofilm volume fraction, and small diffusivity ratio. In general, it can be concluded that the dimensionless coefficient $\xi^{*}$ is insensitive to Péclet number for typical flow conditions in porous media, particularly when $P e^{\mathrm{w}}<1$. The first row of Fig. 5 shows the dependencies of $\xi^{*}$ on Damköhler number for different biofilm volume fractions, dimensionless inlet solute concentrations, and diffusivity 

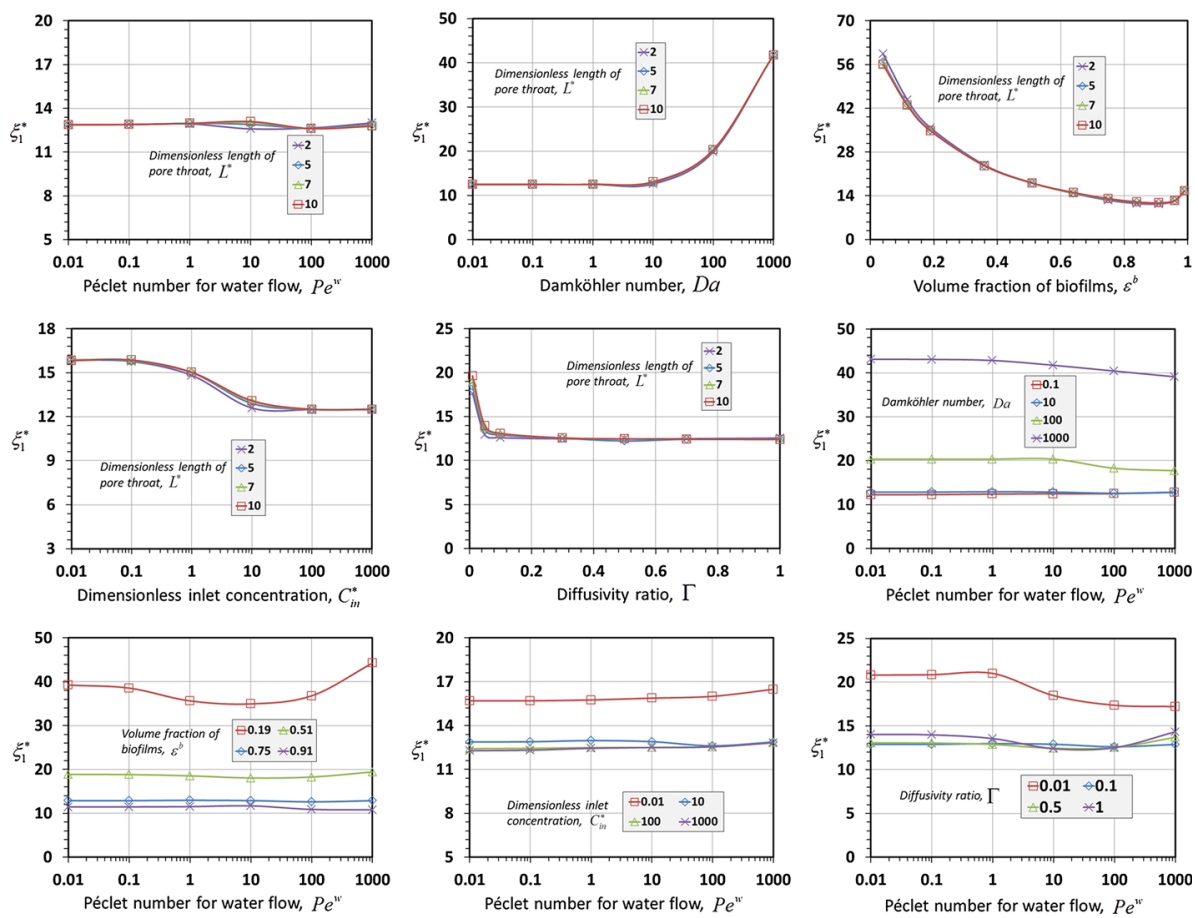

Fig. 4 Dependence of the dimensionless mass exchange coefficient on the length of pore domain and Péclet number

ratios. When $D a<1, \xi^{*}$ can be considered to be independent of Damköhler number. But, beyond this range, $\xi^{*}$ increases with the increase in $D a$. This is because of the increase in the mass exchange rate, while the concentration difference between the water and biofilm does not change much. For a simple unit cell, Golfier et al. (2009) identified the local mass equilibrium regime where Péclet and Damköhler numbers were both smaller than unity. In such case, a simple one-equation model for solute transport can be used. But, when Péclet number is larger than unity, local mass non-equilibrium occurs which requires extra efforts in the modeling (Davit et al. 2010). In the case of using two-equation model with the mass exchange rate, our numerical results show that the dimensionless mass exchange coefficient remains constant when there is no mass-transfer limitation (i.e., for small Damköhler number).

The second row of Fig. 5 shows the dependencies of $\xi^{*}$ on biofilm volume fraction under different operating conditions. Basically, $\xi^{*}$ decreases exponentially with the increase in biofilm volume fraction, except at very high biofilm volume fractions where it increases slightly. In addition, it is seen that the dependencies of $\xi^{*}$ on biofilm volume fraction, inlet solute concentration, and diffusivity ratio are strongly coupled. The third row of Fig. 5 shows the dependencies of $\xi^{*}$ on dimensionless inlet concentration for different biofilm volume fractions, Damköhler numbers, and diffusivity ratios. From the Monod kinetics (see Eq. 1b), it is seen that the bioreaction rate reduces to either first order or zeroth order when the solute concentration is much smaller or much larger than the half-saturation constant, respectively. Therefore, $\xi^{*}$ is expected to be independent of $C_{\text {in }}^{*}$ at very high or low dimensionless inlet solute concentrations. But, due to the strong coupling effect among these dependent variables, the transitional zone between high and low inlet concentrations is enlarged with the increase 

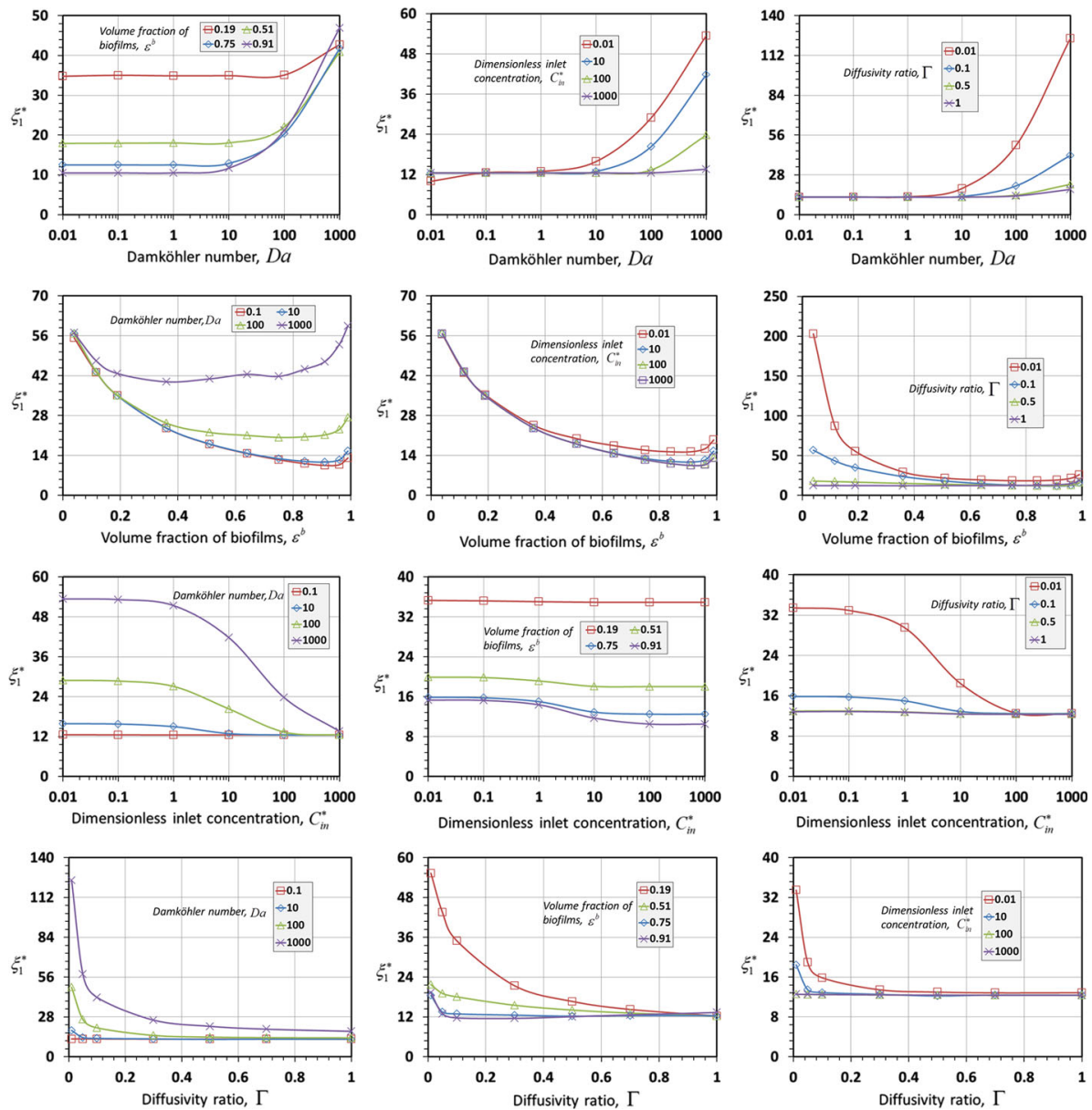

Fig. 5 Dependence of the dimensionless mass exchange coefficient on a Damköhler number, b biofilm volume fraction, $\mathbf{c}$ dimensionless inlet solute concentration, and $\mathbf{d}$ diffusivity ratio

in Damköhler number or diffusivity ratio, and flattened with the decrease of biofilm volume fraction. It is interesting to see that in the regime of zeroth-order bioreaction, $\xi^{*}$ is solely dependent on biofilm volume fraction. Finally, the last row of Fig. 5 shows the dependencies of $\xi^{*}$ on diffusivity ratio. It is seen that $\xi^{*}$ decreases dramatically with the increase in diffusivity ratio when $\Gamma<0.1$. When $\Gamma=1.0$, the dimensionless mass exchange coefficient more or less converges to a constant value of 12 .

With the help of microscale simulations, we have identified the four dimensionless variables that have significant effects on the value of the mass exchange coefficient $\xi^{*}$; they are Damköhler number, biofilm volume fraction, dimensionless inlet solute concentration, and diffusivity ratio. It is found that $\xi^{*}$ increases dramatically under severe mass-transfer limitations, such as at large Damköhler numbers and small diffusivity ratios. Over the past few years, several researchers (Golfier et al. 2009; Hesse et al. 2009; Orgogozo et al. 2010; Wood et al. 2007) have attempted to solve a closure problem of pore-scale concentration fluctuation filed, in order to obtain the effective reaction rate, dispersion tensor, etc. But, each closure 
problem needs to be numerically solved for pre-specified pore structure and flow condition. Alternatively, in this work, we worked on an empirical equation of the mass exchange rate. Based on lots of microscale simulations, in the next section, we will numerically fit empirical formulae which can be directly used in further PNM.

\subsection{Empirical Formulae for the Mass Exchange Coefficient}

As discussed in the previous section, $\xi^{*}$ strongly depends on Damköhler number, biofilm volume fraction, dimensionless inlet solute concentration, and diffusivity ratio. Also, these dependences are intricately coupled. As a result, it is quite difficult to numerically fit the results and obtain a general formula for $\xi^{*}$ as a function of all dependent variables. However, an explicit expression for the dependence of $\xi^{*}$ on various variables is needed for pore-network modeling. To this end, as a first attempt, we have chosen constant values for three parameters. First, we fixed the diffusivity ratio of solute to be 0.5 which is close to experimentally measured values. We notice that for values of diffusivity ratio around 0.5 , the coefficient $\xi^{*}$ does not vary much (see Fig. 5). Also, variation in Péclet number has little effect on the value of $\xi^{*}$, especially in the lower range of $P e^{\mathrm{w}}$ values; so, we set $P e^{\mathrm{w}}=0.1$. Finally, we focused on the condition of mass-transfer limitations and assumed first-order bioreaction to occur in the biofilm. As a result, the input solute concentration does not play a role anymore. With these assumptions, we only need to consider the dependence of $\xi^{*}$ on Damköhler number and biofilm volume fraction. In addition, Damköhler number was constrained between 0.01 and 1000 .

We determined $\xi^{*}$, varying $D a$ and $\varepsilon^{\mathrm{b}}$, for three different cross sections: circle, square, and equilateral triangle. Results are shown in Fig. 6. We then numerically fitted expressions to these results and obtained the following empirical formulae for $\xi^{*}$ :

Circular $\left(R^{2} 0.96\right)$ :

$$
\xi^{*}=\frac{-52.2+0.06 \ln (D a)+18.2 \operatorname{Exp}\left(\varepsilon^{\mathrm{b}}\right)}{1+1.63 \times 10^{-4} D a-0.94 \varepsilon^{\mathrm{b}}}+58.9
$$

Square $\left(R^{2} 0.91\right)$ :

$$
\xi^{*}=\frac{12.7+0.02 D a-12 \varepsilon^{\mathrm{b}}}{1+3.54 \times 10^{-4} D a-0.95 \varepsilon^{\mathrm{b}}}
$$

Equilateral triangular $\left(R^{2} 0.93\right)$ :

$$
\xi^{*}=\frac{12.4+0.03 D a-10.6 \varepsilon^{\mathrm{b}}}{1+4.48 \times 10^{-4} D a-0.93 \varepsilon^{\mathrm{b}}}
$$

\subsection{Solute Transport in a Long Capillary Tube}

In this subsection, verification studies of the proposed mass exchange rate against microscale simulations of solute transport in a long capillary tube with circular cross section are presented. The corresponding dimensionless solute transport equations (53 and 54) in the long tube are presented in "Appendix 3." Taylor-Aris dispersion (Aris 1956) is used for solute transport in the water phase, and first-order bioreaction is considered. Here, three different case studies are conducted with the tube dimensionless length of 50, while the tube diameter taken as the reference length. The employed dimensionless geometric and physical parameters are listed in Table 2. We varied Péclet number, Damköhler number, and biofilm 

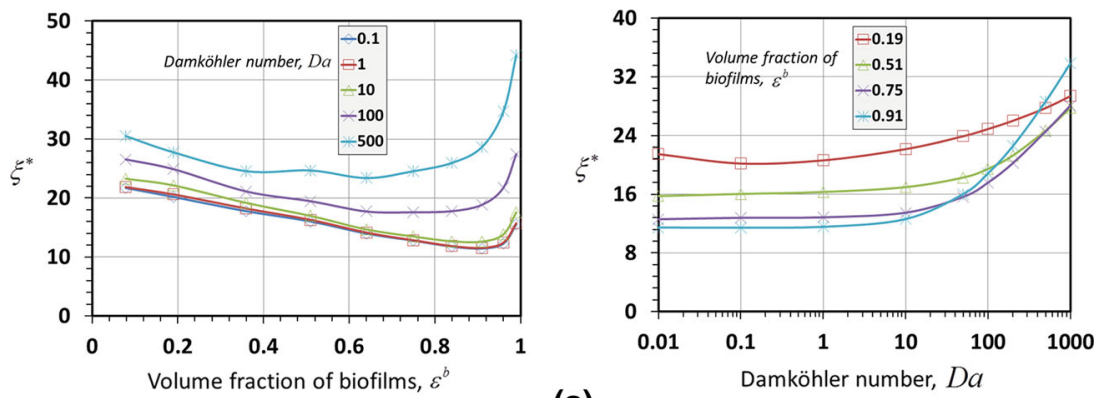

(a)
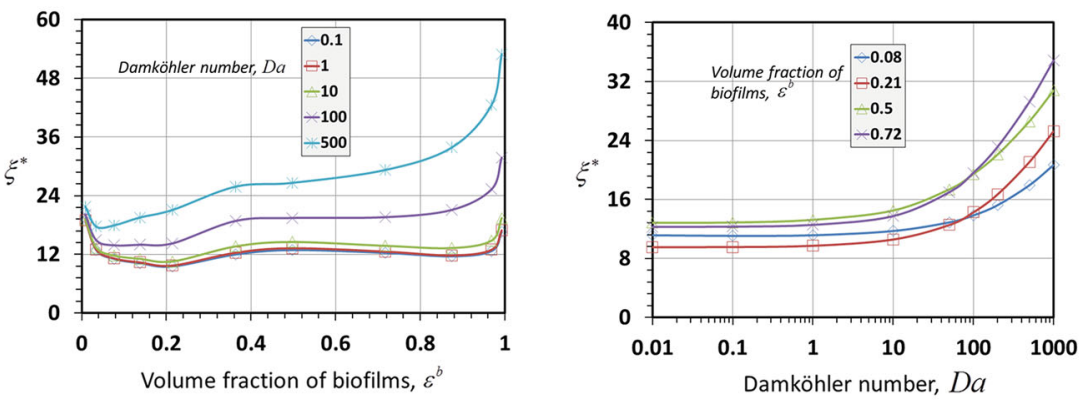

(b)
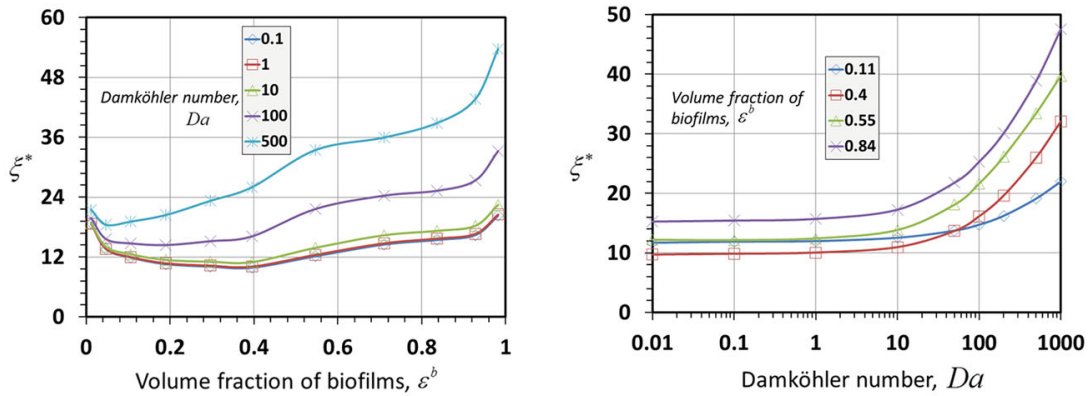

(c)

Fig. 6 Dependence of the dimensionless mass exchange coefficient on biofilm volume fraction and Damköhler number for the cases of a circular cross section, $\mathbf{b}$ square cross section, and $\mathbf{c}$ equilateral triangular cross section, while fixing the remaining variables

volume fraction to represent three typical transport regimes of reaction-rate limited consumption, mass-transfer limited consumption, and an intermediate regime (Orgogozo et al. 2010); we referred to them as case 3, case 2, and case 1, respectively. Because Péclet number, Damköhler number, and biofilm volume fraction are fixed in each case, we can calculate the corresponding specific area and meanwhile obtain the values of dimensionless coefficient $\xi^{*}$ from Eq. 26a. All numerical simulations here were conducted in COMSOL.

Note that for the comparison between the two scales, the results of microscale simulations were averaged over a dimensionless length of 5 along the tube. In case 1, Péclet and Damköhler numbers are 100 and 10, respectively. This represents a general non-equilibrium condition for solute mass exchange between water phase and biofilm (Orgogozo et al. 2010). The comparison of concentration distributions along the tube is shown in Fig. 7a. It is seen that a good 
Table 2 Employed dimensionless geometric and physical parameters for solute transport in a long capillary tube

\begin{tabular}{llll}
\hline Parameter & Value in case 1 & Value in case 2 & Value in case 3 \\
\hline Tube length & 50 & 50 & 50 \\
Tube diameter & 1 & 1 & 1 \\
Péclet number for water flow & 100 & 1 & 1000 \\
Damköhler number & 10 & 1000 & 1000 \\
Diffusivity ratio & 0.5 & 0.5 & 0.5 \\
$\quad$ Volume fraction of biofilms & 0.75 & 0.51 & 0.36 \\
$\begin{array}{l}\text { Mass exchange coefficient obtained from the } \\
\text { database }\end{array}$ & 13.8 & 27.6 & 27.8 \\
$\begin{array}{l}\text { Specific area } \\
\text { nat }\end{array}$ & 2 & 2.8 & 3.2 \\
\hline
\end{tabular}

match was obtained. Also, a good match was obtained for case 2 (i.e., the case of mass-transfer limited consumption). As shown in Fig. 7b, most of solute is immediately consumed at the inlet region due to the high bioreaction rate. When the condition of reaction-rate limited consumption (case 3) was considered, shown in Fig. 7c, a small discrepancy of solute concentration in the water phase was observed. But, the solute concentration in the biofilm was predicted well by the macroscale model. Notice that here we did not present our results in terms of breakthrough curve of solute, mainly because we are more interested in timescales much larger than the resident time in a pore domain when tracking biofilm evolution in porous media.

We have shown that the value of mass exchange coefficient depends on operating conditions. But, in most studies of modeling solute transport between water phase and biofilm, either the mass exchange coefficient has been assumed to be constant or local equilibrium mass transfer has been simply assumed. Fig. 7d shows the sensitivity of solute concentration distributions to the value of mass exchange coefficient, $\xi^{*}$. Here, case 1 is used as the basis with $\xi^{*}$ of 13.8. By increasing the value of $\xi^{*}$, the mass transfer approaches local equilibrium. Otherwise, the mass transfer is away from equilibrium. It is seen that the predicted results are strongly dependent on $\xi^{*}$, and solute concentration in the water phase goes up quickly with decreasing $\xi^{*}$. This sensitivity study indicates that an accurate estimation of the mass exchange coefficient based on operating conditions is crucial to the numerical modeling of solute transport and biofilm growth.

\section{Conclusions and Future Work}

The present work has contributed to describing the solute mass exchange between water phase and biofilm over a single pore domain. This will serve as an important input to the pore-network modeling. We derived a semiempirical equation for the mass exchange rate between water phase and biofilm. A coefficient (usually called mass exchange coefficient) is present as an upscaled property in the mass exchange rate, which is determined by microscale information. To understand the dependencies of this coefficient on a number of variables like Péclet number, Damköhler number, and biofilm volume fraction, we conducted a large number of microscale simulations in a single pore. Three formulae for the mass exchange coefficient were obtained for three types of pore cross section: circle, square, and triangle. Finally, we verified the proposed mass exchange rate against microscale simulations of solute transport in a long capillary tube. Based on the numerical studies in this work, we draw the following main conclusions. In addition, a few words on future work are given below. 

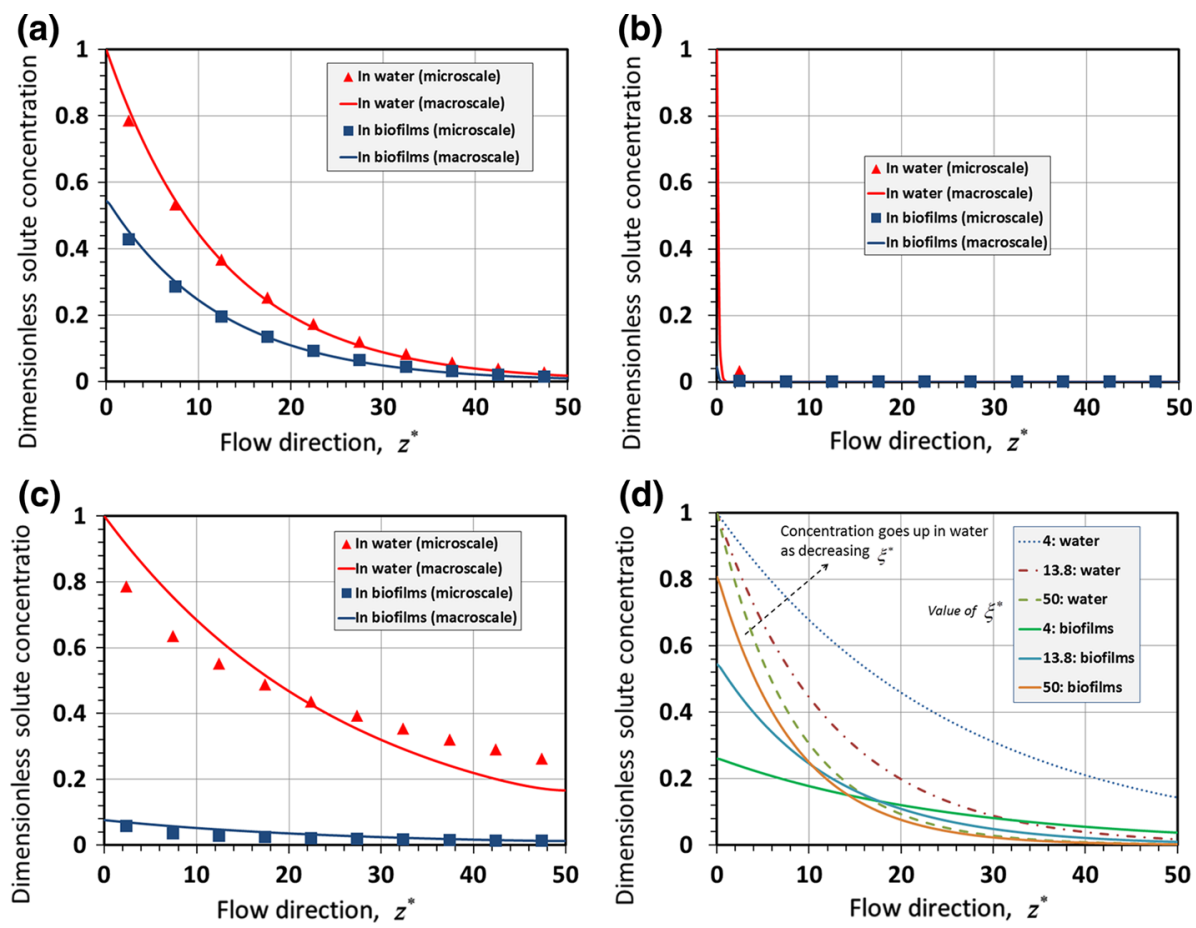

Fig. 7 Comparisons of solute concentration distributions along the capillary tube for a case 1 , b case 2, and c case 3; and $\mathbf{d}$ effect of the mass exchange coefficient on solute concentration distributions

1. For a pore domain with circular cross section, the dimensionless mass exchange coefficient strongly depends on Damköhler number, biofilm volume fraction, dimensionless inlet solute concentration, and solute diffusivity ratio. These dependences are intricately coupled. But, the dimensionless coefficient is independent of pore length and insensitive to Péclet number.

2. By fixing diffusivity ratio and considering small Péclet flow regime and first-order reaction, empirical formulae for the dimensionless mass exchange coefficient were obtained for three types of pore cross section. They are functions of biofilm volume fraction and Damköhler number.

3. In a long capillary tube, the macroscale model with the developed mass exchange rate can predict solute concentration distributions in both water phase and biofilms satisfactorily.

In the near future, the developed mass exchange term with numerically fitted mass exchange coefficient will be used in the pore-network modeling of reactive transport in porous media associated with biofilm growth.

Acknowledgments This research is supported by the Dutch Technology Foundation STW, which is part of the Netherlands Organisation for Scientific Research (NWO) and which is partly funded by the Ministry of Economic Affairs. The authors are members of the International Research Training Group NUPUS, financed by the German Research Foundation (DFG) and The Netherlands Organisation for Scientific Research (NWO).

Open Access This article is distributed under the terms of the Creative Commons Attribution 4.0 International License (http://creativecommons.org/licenses/by/4.0/), which permits unrestricted use, distribution, 
and reproduction in any medium, provided you give appropriate credit to the original author(s) and the source, provide a link to the Creative Commons license, and indicate if changes were made.

\section{Appendix 1}

\section{Derivation of Average-Scale Equations of Solute Transport and Biofilm Growth}

Prior to averaging the microscale transport equations ( $1 \mathrm{a}$ and $1 \mathrm{~b}$ ) over an average domain (e.g., a single pore domain), it is useful to give the following definitions of several important average-scale quantities:

Porosity in the absence of biofilm: $\varepsilon=1-\frac{1}{V} \int_{V^{\mathrm{s}}} \mathrm{d} v$

Volume fraction of biofilm: $\varepsilon^{\mathrm{b}}=\frac{1}{V} \int_{V^{\mathrm{b}}} \mathrm{d} v$

Volume fraction of pore flow: $\varepsilon^{\mathrm{w}}=\varepsilon-\varepsilon^{\mathrm{b}}$

Intrinsic average mass concentration: $\bar{C}_{i}=\frac{1}{V^{i}} \int_{V^{i}} C_{i} \mathrm{~d} v \quad i=w, b$

Intrinsic average water velocity (neglecting density change): $\overline{\mathbf{v}}_{\mathrm{W}}=\frac{1}{V^{\mathrm{w}}} \int_{V^{\mathrm{w}}} \mathbf{v}_{\mathrm{W}} \mathrm{d} v$

Here, $V$ is the porous media volume, the superscript $s$ denotes the solid phase, and $\bar{C}$ is the intrinsic solute concentration. Note that throughout the work, average concentrations are marked with an overbar.

Averaging the microscale transport equation (1a) and employing the spatial and temporal averaging theorems (Pinder and Gray 2008), after some manipulation and algebra, we obtain:

$$
\begin{gathered}
\frac{\partial}{\partial t}\left(\varepsilon^{\mathrm{w}} \bar{C}_{\mathrm{w}}\right)+\nabla \cdot\left(\varepsilon^{\mathrm{w}} \bar{C}_{\mathrm{w}} \overline{\mathbf{v}}_{\mathrm{w}}\right)-\nabla \cdot\left[D _ { \mathrm { w } } \left(\varepsilon^{\mathrm{w}} \nabla \bar{C}_{\mathrm{w}}+\frac{1}{V} \int_{S^{\mathrm{wb}}} C_{\mathrm{w}} \mathbf{n}^{\mathrm{wb}} \mathrm{d} \sigma\right.\right. \\
\left.\left.+\frac{1}{V} \int_{S^{\mathrm{ws}}} C_{\mathrm{w}} \mathbf{n}^{\mathrm{ws}} \mathrm{d} \sigma\right)-\frac{1}{V} \int_{V^{\mathrm{w}}} \tilde{C}_{\mathrm{w}} \tilde{\mathbf{v}}_{\mathrm{w}} \mathrm{d} v\right]=-r_{\mathrm{m}}
\end{gathered}
$$

where

$$
\begin{gathered}
r_{\mathrm{m}}=-D_{\mathrm{w}} \frac{1}{V} \int_{S^{\mathrm{wb}}} \nabla C_{\mathrm{w}} \cdot \mathbf{n}^{\mathrm{wb}} \mathrm{d} \sigma \\
\tilde{C}_{\mathrm{w}}=C_{\mathrm{w}}-\bar{C}_{\mathrm{w}}, \quad \tilde{\mathbf{v}}_{\mathrm{w}}=\mathbf{v}_{\mathrm{w}}-\overline{\mathbf{v}}_{\mathrm{w}}
\end{gathered}
$$

Here, $r_{\mathrm{m}}\left(\mathrm{M} / \mathrm{L}^{3} \mathrm{~T}\right)$ represents the mass exchange rate of solute at the wb interface, which will be positive whenever there is solute consumption in the biofilm. The terms inside angular bracket collectively represent the macroscale dispersion flux. By employing the concept of dispersion tensor in porous media, we can rewrite Eq. 32 as:

$$
\frac{\partial}{\partial t}\left(\varepsilon^{\mathrm{w}} \bar{C}_{\mathrm{w}}\right)+\nabla \cdot\left(\varepsilon^{\mathrm{w}} \bar{C}_{\mathrm{w}} \overline{\mathbf{v}}_{\mathrm{w}}\right)-\nabla \cdot\left(\mathbf{D}_{\mathrm{w}}^{\mathrm{eff}} \nabla \bar{C}_{\mathrm{w}}\right)=-r_{\mathrm{m}}
$$

where $\mathbf{D}_{\mathrm{w}}^{\text {eff }}$ is the effective dispersion tensor for solute transport in the water phase. In the literature, there have been extensive studies on the constitutive relationships for effective dispersion tensor. Recently, for the case of reactive transport and bioreaction, some researchers have derived the closure equations for calculating the effective dispersion tensor under both equilibrium and non-equilibrium conditions (Golfier et al. 2009; Orgogozo et al. 2010). 
Following a similar procedure, Eq. $1 \mathrm{~b}$ for solute transport in the biofilm can be averaged. We assume that the three parameters in Monod kinetics are constant. After some manipulation and algebra, the corresponding average-scale equation is found to be:

$$
\begin{aligned}
\frac{\partial}{\partial t}\left(\varepsilon^{\mathrm{b}} \bar{C}_{\mathrm{b}}\right)-\nabla \cdot\left[D_{\mathrm{b}}\left(\varepsilon^{\mathrm{b}} \nabla \bar{C}_{\mathrm{b}}+\frac{1}{V} \int_{S^{\mathrm{wb}}} C_{\mathrm{b}} \mathbf{n}^{\mathrm{bw}} \mathrm{d} \sigma+\frac{1}{V} \int_{S^{\mathrm{bs}}} C_{\mathrm{b}} \mathbf{n}^{\mathrm{bs}} \mathrm{d} \sigma\right)\right] \\
-D_{\mathrm{b}} \frac{1}{V} \int_{S^{\mathrm{wb}}} \nabla C_{\mathrm{b}} \cdot \mathbf{n}^{\mathrm{bw}} \mathrm{d} \sigma-\frac{1}{V} \int_{S^{\mathrm{wb}}} C_{\mathrm{b}} \mathbf{w} \cdot \mathbf{n}^{\mathrm{bw}} \mathrm{d} \sigma \\
=-k \rho_{\mathrm{b}} \frac{\bar{C}_{\mathrm{b}}}{K_{\mathrm{s}}+\bar{C}_{\mathrm{b}}}\left(\varepsilon^{\mathrm{b}}+\frac{1}{V} \int_{V^{\mathrm{b}}} \frac{K_{\mathrm{s}} \tilde{C}_{\mathrm{b}} / \bar{C}_{\mathrm{b}}}{K_{\mathrm{s}}+\bar{C}_{\mathrm{b}}+\tilde{C}_{\mathrm{b}}} \mathrm{d} V\right)
\end{aligned}
$$

where $\mathbf{n}^{\text {bw }}$ is the normal vector outward from the biofilm. If the interface velocity $\mathbf{w}$ is small enough and the second term in the parenthesis on the r.h.s is negligible in comparison with $\varepsilon^{\mathrm{b}}$ (Hesse et al. 2009; Wood et al. 2007), we obtain:

$$
\frac{\partial}{\partial t}\left(\varepsilon^{\mathrm{b}} \bar{C}_{\mathrm{b}}\right)-\nabla \cdot\left(\mathbf{D}_{\mathrm{b}}^{\mathrm{eff}} \nabla \bar{C}_{\mathrm{b}}\right)-D_{\mathrm{b}} \frac{1}{V} \int_{S^{\mathrm{wb}}} \nabla C_{\mathrm{b}} \cdot \mathbf{n}^{\mathrm{bw}} \mathrm{d} \sigma=-\varepsilon^{\mathrm{b}} k \rho_{\mathrm{b}} \frac{\bar{C}_{\mathrm{b}}}{K_{\mathrm{s}}+\bar{C}_{\mathrm{b}}}
$$

where $\mathbf{D}_{\mathrm{b}}^{\text {eff }}$ is the effective dispersion tensor for solute transport in the biofilm.

As for the biomass evolution, we only consider sessile biofilm attached on the solid walls which is immobile. Its microscale mass balance may be given as:

$$
\frac{\partial \rho_{\mathrm{b}}}{\partial t}=k Y \frac{C_{\mathrm{b}}}{K_{\mathrm{s}}+C_{\mathrm{b}}} \rho_{\mathrm{b}}-r_{\text {decay }}
$$

where $Y$ is the yield coefficient accounting for the fraction of solute actually used for biofilm growth and $r_{\text {decay }}$ is the endogenous decay rate.

Again, we average Eq. 38 to obtain:

$$
\begin{aligned}
\frac{\partial}{\partial t}\left(\varepsilon^{\mathrm{b}} \rho_{\mathrm{b}}\right)-\frac{1}{V} \int_{S^{\mathrm{wb}}} \rho_{\mathrm{b}} \mathbf{w} \cdot \mathbf{n d} \sigma= & k Y \rho_{\mathrm{b}} \frac{\bar{C}_{\mathrm{b}}}{K_{\mathrm{s}}+\bar{C}_{\mathrm{b}}}\left(\varepsilon^{\mathrm{b}}+\frac{1}{V} \int_{V^{\mathrm{b}}} \frac{K_{\mathrm{s}} \tilde{C}_{\mathrm{b}} / \bar{C}_{\mathrm{b}}}{K_{\mathrm{s}}+\bar{C}_{\mathrm{b}}+\tilde{C}_{\mathrm{b}}} \mathrm{d} V\right) \\
& -\varepsilon^{\mathrm{b}} \bar{r}_{\text {decay }}-r_{\text {det }}
\end{aligned}
$$

With the same arguments as in Eqs. 36, 39 can be approximated to the following form:

$$
\rho_{\mathrm{b}} \frac{\partial \varepsilon^{\mathrm{b}}}{\partial t}=\varepsilon^{\mathrm{b}} k Y \frac{\bar{C}_{\mathrm{b}}}{K_{\mathrm{s}}+\bar{C}_{\mathrm{b}}} \rho_{\mathrm{b}}-\varepsilon^{\mathrm{b}} \bar{r}_{\text {decay }}-r_{\text {det }}
$$

Here, the biofilm is assumed to consist of only one type of biomass compound. Its growth is directly related to the degradation of the rate-limiting solute. The biomass density $\rho_{\mathrm{b}}$ is assumed to be constant. In addition, it is noted that we need to introduce the detachment of biomass, $r_{\text {det }}$, as a sink term at the macroscale in Eq. 40. In general, it depends on the shear force exerted on biofilm by water flow. The attachment of planktonic cells is not considered.

\section{Appendix 2}

\section{Dimensionless Microscopic Transport Equations in a Pore with Circular Cross Section}

In the case of a circular cross section, the cylindrical coordinate is used for simplicity (see Fig. 2a). Here, we take the original (without biofilm) diameter of the pore, $d$, as the reference 
length. Then, the following dimensionless variables are defined:

$$
r^{*}=\frac{r}{d}, \quad z^{*}=\frac{z}{d}, \quad L^{*}=\frac{L}{d}, \quad r_{\mathrm{b}}^{*}=\frac{r_{\mathrm{b}}}{d}
$$

So, the biofilm volume fraction can be given as:

$$
\varepsilon^{\mathrm{b}}=\frac{(d / 2)^{2}-r_{\mathrm{b}}^{2}}{(d / 2)^{2}}=1-4 r_{\mathrm{b}}^{* 2}
$$

Based on the Hargen-Poiseuille flow, the conductivity for the circular cross section is expressed as a function of biofilm volume fraction in the following form:

$$
K=\frac{\pi r_{\mathrm{b}}^{4}}{8 \mu}=\frac{\pi d^{4}}{128 \mu}\left(1-\varepsilon^{\mathrm{b}}\right)^{2}
$$

In addition, the average $z$-velocity and the axial distribution of the $z$-velocity are:

$$
\begin{aligned}
& \bar{v}_{\mathrm{z}}=-\frac{\mathrm{d} p}{\mathrm{~d} z} \frac{r_{\mathrm{b}}^{2}}{8 \mu} \\
& v_{\mathrm{z}}=2 \bar{v}_{\mathrm{z}}\left[1-\left(\frac{r}{r_{\mathrm{b}}}\right)^{2}\right]=2 \bar{v}_{\mathrm{z}}\left[1-\left(\frac{r^{*}}{r_{\mathrm{b}}^{*}}\right)^{2}\right]
\end{aligned}
$$

Here, $\mathrm{d} p / \mathrm{d} z$ is the pressure gradient along the flow direction assumed to be constant. The contact area between water flow and biofilm is given by:

$$
A_{\mathrm{wb}}=2 \pi r_{\mathrm{b}} L=\pi d L \sqrt{1-\varepsilon^{\mathrm{b}}}
$$

Now, taking the average $z$-velocity as the reference velocity $\left(v^{\text {ref }}=\bar{v}_{\mathrm{z}}\right)$ and using the defined dimensionless variables listed in (12) and (41), the dimensionless solute transport equations read:

$$
\begin{aligned}
& \frac{\partial C_{\mathrm{w}}^{*}}{\partial t^{*}}+2 P e^{\mathrm{w}}\left[1-{\left.\frac{4\left(r^{*}\right)^{2}}{1-\varepsilon^{\mathrm{b}}}\right]}^{2}\right] \frac{\partial C_{\mathrm{w}}^{*}}{\partial z^{*}}-\left(\frac{\partial^{2} C_{\mathrm{w}}^{*}}{\partial r^{* 2}}+\frac{1}{r^{*}} \frac{\partial C_{\mathrm{w}}^{*}}{\partial r^{*}}+\frac{\partial^{2} C_{\mathrm{w}}^{*}}{\partial z^{* 2}}\right)=0 \\
& \frac{\partial C_{\mathrm{b}}^{*}}{\partial t^{*}}-\Gamma\left(\frac{\partial^{2} C_{\mathrm{b}}^{*}}{\partial r^{* 2}}+\frac{1}{r^{*}} \frac{\partial C_{\mathrm{b}}^{*}}{\partial r^{*}}+\frac{\partial^{2} C_{\mathrm{b}}^{*}}{\partial z^{* 2}}\right)+D a \frac{C_{\mathrm{b}}^{*}}{1+C_{\mathrm{b}}^{*}}=0
\end{aligned}
$$

Here, for solute transport in the water flow, we neglect the r-direction velocity.

\section{Appendix 3}

\section{Dimensionless Governing Equations for Solute Transport in a Long Capillary Tube}

According to the solute transport equations ( $2 \mathrm{a}$ and $2 \mathrm{~b}$ ) and mass exchange rate given by Eq. 9, we can write one-dimensional solute transport equations in a long capillary tube with biofilm in the following form: 


$$
\begin{aligned}
& \frac{\partial}{\partial t}\left(\varepsilon^{\mathrm{w}} \bar{C}_{\mathrm{w}}\right)+\frac{\partial}{\partial z}\left(\varepsilon^{\mathrm{w}} \bar{C}_{\mathrm{w}} \bar{v}_{\mathrm{z}}\right)-\frac{\partial}{\partial z}\left(D_{\mathrm{w}}^{\mathrm{eff}} \frac{\partial \bar{C}_{\mathrm{w}}}{\partial z}\right)=-\frac{D_{\mathrm{w}} D_{\mathrm{b}}}{D_{\mathrm{w}}+D_{\mathrm{b}}} \gamma \xi_{1}\left(\bar{C}_{\mathrm{w}}-\bar{C}_{\mathrm{b}}\right) \\
& \frac{\partial}{\partial t}\left(\varepsilon^{\mathrm{b}} \bar{C}_{\mathrm{b}}\right)-\frac{\partial}{\partial z}\left(D_{\mathrm{b}}^{\mathrm{eff}} \frac{\partial \bar{C}_{\mathrm{b}}}{\partial z}\right)=\frac{D_{\mathrm{w}} D_{\mathrm{b}}}{D_{\mathrm{w}}+D_{\mathrm{b}}} \gamma \xi_{1}\left(\bar{C}_{\mathrm{w}}-\bar{C}_{\mathrm{b}}\right)-\frac{\varepsilon^{\mathrm{b}} k \rho_{\mathrm{b}}}{K_{\mathrm{s}}} \bar{C}_{\mathrm{b}}
\end{aligned}
$$

where $\mathrm{z}$ is the flow direction, $D_{\mathrm{w}}^{\text {eff }}$ and $D_{\mathrm{b}}^{\text {eff }}$ are the effective dispersivities in the water phase and biofilm, respectively, and $\bar{C}_{\mathrm{w}}$ and $\bar{C}_{\mathrm{b}}$ are the average solute concentrations in the water phase and biofilm, respectively. According to the assumption of no advection in biofilm, we may assume that $D_{\mathrm{b}}^{\text {eff }}=\varepsilon^{\mathrm{b}} D_{\mathrm{b}}$. Also, Monod kinetics reduces to the first-order reaction, assuming that the inlet solute concentration is much lower than the half-saturation constant.

To dimensionalize the above transport equations, we define the following dimensionless variables:

$$
\begin{aligned}
\bar{C}_{\mathrm{w}}^{*} & =\frac{\bar{C}_{\mathrm{w}}}{C_{\mathrm{in}}}, \quad \bar{C}_{\mathrm{b}}^{*}=\frac{\bar{C}_{\mathrm{b}}}{C_{\mathrm{in}}}, \quad z^{*}=\frac{z}{L^{\mathrm{ref}}}, \\
t^{*} & =\frac{D_{\mathrm{w}} t}{\left(L^{\text {ref }}\right)^{2}}, \quad P e^{\mathrm{w}}=\frac{\bar{v}_{\mathrm{z}} L^{\mathrm{ref}}}{D_{\mathrm{w}}}, \quad D a=\frac{k \rho_{\mathrm{b}}}{K_{\mathrm{s}} D_{\mathrm{w}}}\left(L^{\mathrm{ref}}\right)^{2}
\end{aligned}
$$

In addition, when considering a tube with circular cross section, taking the tube diameter as the reference length, and assuming fully developed flow, Taylor-Aris dispersion may be used in the water phase, which reads (Aris 1956):

$$
D_{\mathrm{w}}^{\mathrm{eff}}=\varepsilon^{\mathrm{w}} D_{\mathrm{w}}\left[1+\frac{\varepsilon^{\mathrm{w}}}{192}\left(P e^{\mathrm{w}}\right)^{2}\right]
$$

Finally, substitution of dimensionless variables (51) and Eq. 52 into Eq. 49 and Eq. 50 yields the following dimensionless solute transport equations:

$$
\begin{gathered}
\frac{\partial}{\partial t^{*}}\left(\varepsilon^{\mathrm{w}} \bar{C}_{\mathrm{w}}^{*}\right)+P e^{\mathrm{w}} \frac{\partial}{\partial z^{*}}\left(\varepsilon^{\mathrm{w}} \bar{C}_{\mathrm{w}}^{*}\right)-\left[1+\frac{\varepsilon^{\mathrm{w}}}{192}\left(P e^{\mathrm{w}}\right)^{2}\right] \frac{\partial}{\partial z^{*}}\left(\varepsilon^{\mathrm{w}} \frac{\partial C_{\mathrm{w}}^{*}}{\partial z^{*}}\right) \\
=-\frac{\Gamma}{1+\Gamma} \gamma^{*} \xi_{1}^{*}\left(\bar{C}_{\mathrm{w}}^{*}-\bar{C}_{\mathrm{b}}^{*}\right) \\
\frac{\partial}{\partial t^{*}}\left(\varepsilon^{\mathrm{b}} \bar{C}_{\mathrm{b}}^{*}\right)-\Gamma \frac{\partial}{\partial z^{*}}\left(\varepsilon^{\mathrm{b}} \frac{\partial \bar{C}_{\mathrm{b}}^{*}}{\partial z^{*}}\right)=\frac{\Gamma}{1+\Gamma} \gamma^{*} \xi_{1}^{*}\left(\bar{C}_{\mathrm{w}}^{*}-\bar{C}_{\mathrm{b}}^{*}\right)-D a \varepsilon^{\mathrm{b}} \bar{C}_{\mathrm{b}}^{*}
\end{gathered}
$$

\section{References}

Afrapoli, M.S., Alipour, S., Torsaeter, O.: Fundamental study of pore scale mechanisms in microbial improved oil recovery processes. Transp. Porous Media 90, 949-964 (2011)

Aris, R.: On the dispersion of a solute in a fluid flowing through a tube. Proc. R. Soc. Lond. A (1956). doi:10. 1098/rspa.1956.0065

Baveye, P., Valocchi, A.: An evaluation of mathematical models of the transport of biologically reacting solutes in saturated soils and aquifers. Water Resour. Res. 25(6), 1413-1421 (1989)

Baveye, P., Vandevivere, P., Hoyle, B.L., DeLeo, P.C., Sanchez de Lozada, D.: Environmental impact and mechanisms of the biological clogging of saturated soils and aquifer materials. Crit. Rev. Environ. Sci. Technol. 28(2), 123-191 (1998)

Budek, A., Szymczak, P.: Network models of dissolution of porous media. Phys. Rev. E (2012). doi:10.1103/ PhysRevE.86.056318

Cunningham, A.B., Characklis, W.G., Abedeen, F., Crawford, D.: Influence of biofilm accumulation on porous media hydrodynamics. Environ. Sci. Technol. 25(7), 1305-1311 (1991)

Cunningham, J.A., Mendoza-Sanchez, I.: Equivalence of two models for biodegradation during contamination transport in groundwater. Water Resour. Res. (2006). doi:10.1029/2005WR004205 
Davit, Y., Debenest, G., Wood, B.D., Quintard, M.: Modeling non-equilibrium mass transport in biologically reactive porous media. Adv. Water Resour. 33, 1075-1093 (2010)

Dupin, H.J., Kitanidis, P.K., McCarty, P.L.: Simulations of two-dimensional modeling of biomass aggregate growth in network models. Water Resour. Res. 37(12), 2981-2994 (2001)

Ebigbo, A., Helmig, R., Cunningham, A.B., Class, H., Gerlach, R.: Modeling biofilm growth in the presence of carbon dioxide and water flow in the subsurface. Adv. Water Resour. 33, 762-781 (2010)

Ebigbo, A., Philips, A., Gerlach, R., Helmig, R., Cunningham, A.B., Class, H., Spangler, L.H.: Darcy-scale modeling of microbially induced carbonate mineral precipitation in sand columns. Water Resour. Res. (2012). doi:10.1029/2011WR011714

Ezeuko, C.C., Sen, A., Grigoryan, A., Gates, I.D.: Pore-network modeling of biofilm evolution in porous media. Biotechnol. Bioeng. 108(10), 2413-2423 (2011)

Golfier, F., Wood, B.D., Orgogozo, L., Quintard, M., Bues, M.: Biofilms in porous media: development of macroscopic transport equations via volume averaging with closure for local mass equilibrium conditions. Adv. Water Resour. 32, 463-485 (2009)

Graf von der Schulenburg, D.A., Pintelon, T.R.R., Picioreanu, C., Van Loosdrecht, M.C.M., Johns, M.L.: Three-dimensional simulations of biofilm growth in porous media. AIChE J. 55(2), 494-504 (2009)

Hassanizadeh, S.M., Gray, W.G.: General conservation equations for multi-phase systems: 1. Averaging procedure. Adv. Water Resour. 2, 131-144 (1979)

Hesse, F., Radu, F.A., Thullner, M., Attinger, S.: Upscaling of the advection-diffusion-reaction equation with Monod reaction. Adv. Water Resour. 32, 1336-1351 (2009)

Iltis, G.C., Armstrong, R.T., Jansik, D.P., Wood, B.D., Wildenschild, D.: Imaging biofilm architecture within porous media using synchrotron-based X-ray computed microtomography. Water Resour. Res. (2011). doi:10.1029/2010WR009410

Kapellos, G.E., Alexiou, T.S., Payatakes, A.C.: Hierarchical simulator of biofilm growth and dynamics in granular porous materials. Adv. Water Resour. 30, 1648-1667 (2007)

Kim, D.S., Fogler, H.S.: Biomass evolution in porous media and its effects on permeability under starvation conditions. Biotechnol. Bioeng. 69(1), 47-56 (2000)

Li, L., Peters, C.A., Celia, M.A.: Upscaling geochemical reaction rates using pore-scale network modeling. Adv. Water Resour. 29, 1351-1370 (2006)

MacDonald, T.R., Kitanidis, P.K., McCarty, P.L., Roberts, P.V.: Mass-transfer limitations for macroscale bioremediation modeling and implications on aquifer clogging. Ground Water 37(4), 523-531 (1999)

Mitchell, A.C., Phillips, A.J., Hiebert, R., Gerlach, R., Spangler, L.H., Cunningham, A.B.: Biofilm enhanced geologic sequestration of supercritical $\mathrm{CO}_{2}$. Int. J. Greenh. Gas Control 3, 90-99 (2009)

Monod, J.: The growth of bacterial cultures. Annu. Rev. Microbiol. 3, 371-394 (1949)

Orgogozo, L., Golfier, F., Bues, M., Quintard, M.: Upscaling of transport processes in porous media with biofilms in non-equilibrium conditions. Adv. Water Resour. 33, 585-600 (2010)

Pinder, G.F., Gray, W.G.: Essentials of Multiphase Flow and Transport in Porous Media. Wiley, Hoboken (2008)

Pintelon, T.R.R., Graf von der Schulenburg, D.A., Johns, M.L.: Towards optimum permeability reduction in porous media using biofilm growth simulations. Biotechnol. Bioeng. 103(4), 767-779 (2009)

Raoof, A., Hassanizadeh, S.M.: Upscaling transport of adsorbing solutes in porous media. J. Porous Media 13(5), 395-408 (2010)

Raoof, A., Hassanizadeh, S.M., Leijnse, A.: Upscaling transport of adsorbing solutes in porous media: porenetwork modeling. Vadose Zone J. 9, 624-636 (2010)

Rittmann, B.E.: The significance of biofilms in porous media. Water Resour. Res. 29(7), 2195-2202 (1993)

Stewart, T.L., Kim, D.S.: Modeling of biomass-plug development and propagation in porous media. Biochem. Eng. J. 17, 107-119 (2004)

Suchomel, B.J., Chen, B.M., Allen, M.B.: Network model of flow, transport and biofilm effects in porous media. Transp. Porous Media 30, 1-23 (1998a)

Suchomel, B.J., Chen, B.M., Allen, M.B.: Macroscale properties of porous media from a network model of biofilm processes. Transp. Porous Media 31, 39-66 (1998b)

Taylor, S.W., Jaffé, P.R.: Biofilm growth and the related changes in the physical-properties of a porous medium. 3. Dispersivity and model verification. Water Resour. Res. 26(9), 2171-2180 (1990a)

Taylor, S.W., Jaffé, P.R.: Substrate biomass transport in a porous medium. Water Resour. Res. 26(9), 2181-2194 (1990b)

Thullner, M., Zeyer, J., Kinzelbach, W.: Influence of microbial growth on hydraulic properties of pore networks. Transp. Porous Media 49, 99-122 (2002)

Thullner, M., Schroth, M.H., Zeyer, J., Kinzelbach, W.: Modeling of a microbial growth experiment with bioclogging in a two-dimensional saturated porous media flow field. J. Contam. Hydrol. 70, 37-62 (2004) 
Thullner, M., Baveye, P.: Computational pore network modeling of the influence of biofilms permeability on bioclogging in porous media. Biotechnol. Bioeng. 99(6), 1337-1351 (2008)

van Noorden, T.L., Pop, I.S., Ebigbo, A., Helmig, R.: An upscaled model for biofilm growth in a thin strip. Water Resour. Res. (2010). doi:10.1029/2009WR008217

Videla, H.A., Herrera, L.K.: Understanding microbial inhibition of corrosion. A comprehensive overview. Int. Biodeterior. Biodegradation 63, 896-900 (2009)

Wood, B.D., Whitaker, S.: Diffusion and reaction in biofilms. Chem. Eng. Sci. 53, 397-425 (1998)

Wood, B.D., Whitaker, S.: Multi-species diffusion and reaction in biofilms and cellular media. Chem. Eng. Sci. 55, 3397-3418 (2000)

Wood, B.D., Radakovich, K., Golfier, F.: Effective reaction at a fluid-solid interface: applications to biotransformation in porous media. Adv. Water Resour. 30, 1630-1647 (2007)

Zuo, R.J.: Biofilms: strategies for metal corrosion inhibition employing microorganisms. Appl. Microbiol. Biotechnol. 76, 1245-1253 (2007) 